\title{
Tangence
}

\section{Anne Dandurand et Claire Dé : gémellité et passion}

\section{Kathleen Kellett-Betsos}

Numéro 62, avril 2000

Parentèle

URI : https://id.erudit.org/iderudit/008174ar

DOI : https://doi.org/10.7202/008174ar

Aller au sommaire du numéro

Éditeur(s)

Presses de l'Université du Québec

ISSN

0226-9554 (imprimé)

1710-0305 (numérique)

Découvrir la revue

Citer cet article

Kellett-Betsos, K. (2000). Anne Dandurand et Claire Dé : gémellité et passion.

Tangence, (62), 73-83. https://doi.org/10.7202/008174ar d'utilisation que vous pouvez consulter en ligne.

https://apropos.erudit.org/fr/usagers/politique-dutilisation/ 


\title{
Anne Dandurand et Claire Dé : gémellité et passion
}

\author{
Kathleen Kellett-Betsos, \\ Ryerson Polytechnical University
}

Selon la note biographique de son recueil L'assassin de l'intérieur/Diables d'espoir, "Anne Dandurand n'a eu aucun effort à déployer pour venir à la vie, puisque sa jumelle Claire Dé lui a ouvert le chemin, l'année de la naissance de la télévision à Montréal", cette année étant 1953. Pour Claire Dé, leur date de naissance c'est "le 30 Brumaire de l'an révolutionnaire 162", mais elle ne néglige pas, elle non plus, de mentionner sur la couverture de ses livres sa jumelle Anne Dandurand ${ }^{1}$. Ce rapport de parenté porte à conséquence pour la réception de leurs œuvres d'autant plus que les sœurs mènent des carrières littéraires parallèles. Dé, qui détient un diplôme en journalisme de l'Université de Montréal, s'est distinguée dans le théâtre comme conceptrice de décors mais aussi comme traductrice et dramaturge ${ }^{2}$. De son côté, Dandurand a travaillé comme comédienne, syndicaliste, journaliste, scénariste et cinéaste. Les deux ont écrit ensemble la série détective pour la jeunesse $R a$ chel et Réjean, diffusée à la télévision de Radio-Canada en 1987. En 1989, Dé a gagné le Prix Stendhal de la nouvelle pour le recueil Le désir comme catastrophe naturelle alors que la même année Dandurand s'est vue décerner le Grand Prix de la nouvelle pour la jeunesse pour "La dernière journée du milk-shake ${ }^{3}$. Dandurand vit à Montréal tandis que Dé est partie vivre à Paris au début des années quatre-vingt, ce qui explique en partie le cadre hexagonal de certains de ses écrits. Elles ont donc expérimenté plusieurs genres littéraires, mais elles sont surtout reconnues en tant que nouvellistes,

1 L'assassin de l'intérieur / Diables d'espoir, Montréal, XYZ éditeur, 1988. La citation se trouve dans la moitié du recueil intitulée Diables d'espoir, p. 65. Il est à noter que les deux parties du recueil sont reliées l'une à l'autre, certes, mais à l'envers, et paginées séparément. Voir aussi la couverture des livres de Dé, Le désir comme catastrophe naturelle, Montréal-Paris, l'Étincelle, 1989, et Chiens divers (et autres faits écrasés), Montréal, XYZ éditeur, 1991.

2 Quatre de ses pièces se retrouvent dans son livre Sentimental à l'os, Montréal, VLB éditeur, 1991.

3 Petites âmes sous ultimatum, Montréal, XYZ éditeur, 1991. Voir note, p. 110. 
74

s'inscrivant dans une appréciation renouvelée de ce genre littéraire, au Québec, depuis les dernières décennies.

L'essor de la nouvelle au Québec est marqué par la création dans les années 1980 de la revue $X Y Z$ qui lui est consacrée, ainsi que de la maison d'édition XYZ éditeur qui a publié la plupart des ouvres de Dé et de Dandurand. Rares sont les anthologies de nouvelles québécoises qui n'incluent pas un texte de l'une ou l'autre sour sinon des deux. Un exemple notable se trouve dans Qui a peur de...? parrainé par la revue féministe La vie en rose, où chaque auteure écrit une nouvelle inspirée par une écrivaine. Dandurand se situe nettement dans l'Amérique moderne, imaginant dans "Merveilleuse allergie" un fantasme érotique et humoristique digne de l'auteure américaine Erica Jong. Par contre, dans "Encore une tentative...", Dé, qui a également adapté pour la scène la correspondance de George Sand et Alfred de Musset ${ }^{4}$, présente une rencontre avec George Sand dans un café du $\mathrm{XIX}^{\mathrm{e}}$ siècle ${ }^{5}$. Ensemble, Dé et Dandurand ont défriché le terrain du fantastique érotique et féministe dans leur recueil de nouvelles La louve-garou ${ }^{6}$, qui comprend douze récits de Dé et onze de Dandurand. L'insistance sur leur identité de jumelles se manifeste sur la couverture avec une photo des deux suivie à l'intérieur d'une encre de Micheline Rouillard qui représente leurs deux visages opposés, soudés par une chevelure abondante. La critique réagit à ce recueil en essayant généralement de différencier leur écriture. Gilles Cossette, par exemple, conclut ainsi son compterendu: "Claire Dé écrivaine et l'écrivaine Anne Dandurand, on le voit, sont loin d'être identiques ${ }^{7}$..." Selon Claude Janelle, "[...] rien à prime abord ne distingue les textes de Claire Dé de ceux d'Anne Dandurand. Mais ils sont pourtant différents, non pas au niveau de l'inspiration mais dans leur approche et dans leur tona-

4 "Les Amours de George et d'Alfred" (1986). Voir le Théâtre québécois: ses auteurs, ses pièces: répertoire du Centre d'essai des auteurs dramatiques, Montréal, VLB éditeur/Centre d'essai des auteurs dramatiques, édition revue et corrigée, 1990, p. 59-61.

5 "Merveilleuse allergie" et "Encore une tentative", dans Collectif, Qui a peur de?.. (une idée de La Vie en Rose), Montréal, VLB éditeur, 1987, p. 83-88 et 125-130.

6 La louve-garou, Montréal, La Pleine Lune, 1982. Les références aux textes sont les suivantes: $L G$ pour La louve-garou, Désir pour Le désir comme catastrophe naturelle, et Chiens pour Chiens divers (et autres faits écrasés).

7 Gilles Cossette, "Transports en commun", Lettres québécoises, Montréal, $\mathrm{n}^{\mathrm{O}} 27$, automne 1982, p. 28. 
lité». Selon lui, Dé se caractérise par un humour optimiste et parodique; Dandurand, par un ton sérieux et plutôt réaliste ${ }^{8}$. En "interviewant" sa sour pour la revue $X Y Z$, Dé affirme: "Moi j'écris avec elle (et bonne chance à nos futurs biographes pour, dans certains cas, démêler ce que nous avons respectivement créé) ${ }^{9}$ ". Loin de nous l'idée de relever un tel défi! Prenons plutôt plaisir à lire la nouvelle chez ces deux nouvellistes apparentées mais non identiques à travers trois courants annoncés dès $L a$ louve-garou: le fantastique, l'érotisme, et le crime passionnel.

\section{Le fantastique au féminin}

Dans leur fascination pour le fantastique, Dandurand et Dé participent d'une longue tradition québécoise. Le titre même de La louve-garou renvoie à un corpus considérable de récits québécois de loups-garous écrits par des auteurs fondateurs dont Honoré Beaugrand, Pamphile Lemay et Louis Fréchette ${ }^{10}$. Dans "Le désir comme catastrophe naturelle", Dé évoque explicitement l'héritage du conte fantastique québécois en identifiant l'homme désiré comme le diable en personne: «[...] n'avais-je pas lu, ou entendu, ces vieilles légendes québécoises où Satan a tant dansé que le plancher en est resté marqué? [...] J'étais prête à me lancer dans le vide, jouer avec le feu, perdre la raison ou la vie parce que ce diable-là m'avait toute retournée" (Désir, p. 15). Dans "L'évolution de la nouvelle au Québec", Patrick Coleman voit la prépondérance du fantastique dans la nouvelle québécoise moderne comme "un retour de l'imaginaire évacué par la nouvelle dans sa lutte avec le conte", "un instrument pour réintégrer l'imaginaire dans le monde, et dans l'œuvre ${ }^{11}$ ". Certainement, on retrouve une part de nostalgie sous-jacente au fantastique chez ces deux auteures. Dans "L'insémination du ciel", paru dans Petites âmes sous ultimatum de Dandurand, mais publié en une version ultérieure signée des deux sœurs comme "Un journal de spore ${ }^{12}$,

8 Claude Janelle, "Érotisme et féminisme dans le fantastique", Solaris, Montréal, vol. 8, n 6, novembre-décembre 1982, p. 8.

9 "Écrire ou mourir", XYZ, Montréal, no 12, hiver 1987, p. 37.

10 Voir Jeanne Demers et Lise Gauvin, "Frontières du conte écrit - quelques loups-garous québécois", Littérature, Paris, n 45, février 1982, p. 5-23.

11 Patrick Coleman, "L'évolution de la nouvelle au Québec", XYZ, Montréal, no 10, été 1987, p. 68.

12 Voir note Petites âmes..., p. 109. La première version a paru dans L'année de la science-fiction et du fantastique québécois, Beauport, le Passeur, 1989. 
il s'agit d'un être humain subissant graduellement la transformation en spore, ce qui lui permet de se distancier des maux du vingtième siècle: "J'ai si soif, je bois de l'eau sans arrêt, je prends des douches plusieurs fois par jour. J'éprouve un étrange détachement envers tout ce qui me bouleversait, les tueries injustes, les violences médiatisées ou intimes, le manque d'amour... Que m'arrive-t-il?" (p. 42). La narratrice du "Désir comme catastrophe naturelle" avoue ne pas avoir reconnu à première vue le diable pour qui elle aura le coup de foudre, puisque "comme tout rejeton de ce siècle qui a engendré les chambres à gaz, les massacres à la scie, les guerres chimiques et la bombe atomique, je ne croyais ni à Dieu ni à diable" (Désir, p. 13). L'amour idéal n'est ni de ce temps ni de ce monde. Les amants les plus heureux semblent être des créatures de fable, frôlant même le grotesque: par exemple l'histoire d'amour entre le cannibale et l'ogresse dans "Samedi tard" de Claire Dé :

Il l'avale, elle le savoure, les yeux fermés, en claquant la langue, gloutonnerie exquise, gouffre goinfre de douceur, il l'étreint, elle l'absorbe, il la mastique, elle le gobe, il la serre, elle le prend et il la prend. Le tremblement les secoue, la sueur les embrume, leur respiration se creuse, l'indicible extase les aspire par le haut. L'ogresse jouit, le cannibale éjacule (LG, p. 68).

Par contre, le désir inassouvi dans "L'autopsie de la papillonne" de Dandurand tisse autour de l'héroine "le cocon terrible de l'attente" ( $L G$, p. 79$)$ et sa transformation en papillonne à la recherche de l'amour mène à la mort.

Chez Dandurand et Dé, le fantastique sert à soutenir une vision toute féminine de l'amour, de l'érotisme et du pouvoir. Dans "Un cas de lycanthropie" par Dé, une femme, qui "avait oublié qu'il est interdit de se promener sur la rue, à la noirceur, seule et femme" ( $L G$, p. 20) est attaquée par un inconnu mais se venge en se transformant en homarde gigantesque qui écrase l'homme de ses pinces énormes: "Le visage de l'inconnu se révulsa de terreur. Ce fut son dernier sentiment, la pince le coupa au raz le menton avec un crac de casse-noisettes. L'autre pince, la droite, le disloqua en deux, dans le sens de la longueur, à partir du sexe" (p. 21). Les pouvoirs fantastiques accordent aussi à la femme désirante la force de s'emparer de l'objet du désir. Dans "Home sweet home" de Dandurand, une femme meurt pour revenir hanter la maison de l'homme qu'elle désire, le faisant chuter dans la folie, délaissé par la femme qu'il aime, pour enfin le consommer 
dans les flammes de la maison en feu $(L G)$. La sorcière Jeanne Couteau, un personnage introduit dans La louve-garou mais qui revient souvent chez Dandurand, est complice de la narratrice. Dans "Pour endormir ma mort", la narratrice fait l'amour à son amant endormi et ensorcelé: "Mon amant dort, bien enclavé dans mes bras. J'ai appris un nouveau tour de Jeanne Couteau, la sorcière. Il me faut ton sommeil profond, mon bel amour, un sommeil de mort, si je veux bien réussir mon coup ${ }^{13}$." Le fantastique accorde à la femme du pouvoir sur les hommes, pour renverser la domination patriarcale.

Ce désir de dominer les hommes et même de se venger d'eux fait partie de ce que Luise von Flotow appelle "confrontational or conflictual erotica ${ }^{14}$ " chez Dandurand et Dé. L'exemple le plus frappant cité par von Flotow est "L'histoire de Q" par Dandurand, publiée pour la première fois dans La vie en rose et qui a suscité une discussion animée sur la frontière entre érotisme et pornographie. Dans cette nouvelle, une femme fait enlever par ses amies l'amant coupable de ne pas l'aimer assez. Elles font subir à l'homme une torture érotique, le réduisant à l'objet du désir, soumis et sans défense. À la fin, pénitent et conscient de son injustice envers la femme qui l'aime, il la retrouve: "Elle se secoue, le délivre, lui dit: pars. Il refuse. Il dit: laisse-moi mon masque et mes chaînes, ce sont les signes de l'amour. Elle se couche alors sur lui, et commence à le dévorer" (Voilà, p. 29-30). Chez Dé, la scène érotique paraît moins conflictuelle mais on remarque dans "Toujours la nuit" que la femme revendique un statut égal sinon supérieur à l'homme:

Elle lève sur lui ses yeux luisants. Le contemplerait, penché sur elle. Bandé. Le désir de lui plus fort que tout, soudain. Qu'elle se serait brandie vers lui. Qu'elle quêterait son corps. Ses lèvres. L'homme et la femme se seraient enlacés. Comme deux lutteurs. Et auraient lutté.

— Tu gagnes. Tu gagnes lui aurait enfin dit l'homme. Les épaules collées au matelas (Désir, p. 142).

13 Voilà c'est moi c'est rien j'angoisse (journal imaginaire), Montréal, Triptyque, 1987, p. 13.

14 Luise Von Flotow, "Tenter l'érotique... Eroticism in Contemporary Women's Writing from Quebec", Québec Studies, printemps-été 1990, p. 92; voir aussi de la même, "Women's Desiring Voices from Quebec: Nicole Brossard, Anne Dandurand and Claire Dé", dans Us/Them: Translation, Transcription and Identity in Post-Colonial Literary Cultures, Amsterdam, Rodopi, 1992, p. 109-119. 
78

Von Flotow remarque aussi que l'emploi fréquent du conditionnel et l'évocation d'une atmosphère de conte de fées ou de rêve chez Dandurand et Dé pourraient servir à déjouer une interprétation littérale, atténuant ainsi la responsabilité de l'auteur pour l'érotisme explicite et parfois violent ${ }^{15}$. Ajoutons que le recours au fantastique et au grotesque joue le même rôle de distanciation, si l'on pense, par exemple, à l'amour entre l'ogresse et le cannibale dans "Samedi tard". On pourrait dire, dans une optique plus positive, que la mise en relief de la littérarité de la nouvelle érotique représente la valorisation du processus de création et de l'écriture même.

Chez les deux auteures, l'expression de l'érotisme passe par le ludique. On pense, par exemple, à "Une orgie costumée" de Dé où la narratrice s'interroge sur ce qu'il faudrait porter pour une telle affaire (Chiens). Remarquons aussi certains passages légers, comme cette réflexion de "Marc-André, sa blonde et Blanche" de Dandurand: "Avec elles deux j'ai eu l'impression de léviter, et d'éjaculer comme à treize ans, les premières fois. Je ne suis pas certain de ne pas avoir vraiment flotté dans les airs, mais je m'en fous, il faut rêver une fois comme ça avant de mourir" (Voilà, p. 36). D'ailleurs, c'est un amant serviable qui se plaît à être "ensorcelé" par sa blonde Jeanne: "Elle me répète qu'elle a besoin de baiser souvent pour ne pas exploser. Je m'exécute, vous pensez bien. TOUS les soirs, même malade" (Voilà, p. 34). Dans "Les dessous" de Dé, le narrateur et son amante discutent tout amicalement de l'importance du choix des sous-vêtements: "Mon cher amant, c'est parce que le caleçon fait l'homme... que je refuse de lui en procurer. C'est par ses sous-vêtements que l'on juge l'individu. La petite culotte dévoile tout" ( $L G$, p. 92). La guerre des sexes n'est donc pas sans connaitre ses moments de trêve.

Le discours érotique peut atteindre aussi un fort degré de sensualité comme dans "Victime des pénombres où les sens rencontrent le cour" où Dandurand se sert du "journal imaginaire", forme qu'elle affectionne particulièrement, pour donner voix au désir et à l'angoisse de la femme négligée par son amant marié :

La lumière d'un autre matin chatoie dans ses ors et ses rouges, encore une fois tu es venu me voir, et la joie coule en moi

15 Luise von Flotow, "Tenter l'érotique... ", p. 96. 
comme un virus, une drogue, encore une fois tu m'as révélé l'eau la plus pure, la plus parfumée de ton corps de rubis, et pourtant (Diables, p. 15)

L'écoulement des paroles crée une sensualité du langage qui fait écho au sens. Pour Dé aussi, le monologue intérieur joue à véhiculer l'érotisme intime de la femme; dans "la causeuse orientale", par exemple: "Comme il a bien appris, comme il me fait jouir. Comme j'ai soif, ce soir, de ses baisers, de sa bouche, de son corps, de ses mains. Soif de son sexe" ( $L G$, p. 111). Cependant, comme on le verra, le monologue intérieur chez Dé sert plus souvent à communiquer la turbulence du crime passionnel. Chez les deux auteures, la nouvelle érotique communique l'intensité du désir au féminin et revendique le droit à la passion dans un monde hostile à l'amour.

\section{Les avatars du crime passionnel}

Dans le monde féminin évoqué par Dandurand et Dé, la femme est surtout la justicière qui punit les crimes contre l'amour. En bonne journaliste, Dé signale un rapprochement entre ses nouvelles et le fait divers, comme le suggère le titre du recueil Chiens divers (et autres faits écrasés). Par contre, Dandurand privilégie le récit policier. Dans les nouvelles de Dé, la justicière punit la tentative de viol ("Un cas de lycanthropie", $L G$ ), l'abus sexuel de la fille par le père ("Pourquoi les marmottes", Chiens), l'infidélité ("Pot de Colle", Désir). Le récit policier apparaît comme un thème dans "Pot de colle" où un homme, profondément ennuyé de la jalousie de sa femme et de son désir inlassable d'analyser le rapport de couple, commence à l'empoisonner lentement et finit par l'étouffer avec un oreiller. Pourtant, il meurt à son tour, grâce au sabotage de sa voiture par sa femme, tout en écoutant à la radio un texte écrit par celle-ci relatant le meurtre parfait déguisé en accident d'automobile, récit dont elle lui avait pourtant parlé auparavant. Par contre, dans "À tuer" la femme trahie ne peut pas se résoudre à tuer son mari infidèle qui lui refuse son amour et elle se met plutôt à tuer tous les habitants désagréables du quartier avant de tirer sur des policiers, souhaitant être tuée elle-même (Désir). Ces deux nouvelles (et d'autres encore de Dé) se caractérisent par une technique de monologue intérieur fortement saccadé qui communique la passion débridée: "Toi, comme tous les hommes. Lâche. Voulant ménager à la foi la 
80

bique extra-conjugale et le célerirave domestique. Alors ton aventure. Ta mâtresse y a coupé court. Mais. Même après que. Tu as continué. De ne pas me." (Désir, p. 42). Quoique maniacodépressive, cette femme est drôlement consciente des normes de la rectitude politique: "L'Arabe s'affaisse sur sa caisse. Je retire l'Opinel. Pas tellement de sang. [...] J'espère qu'ils ne colporteront pas que c'est un crime raciste. Moi, ce n'est pas les Arabes, c'est l'humanité en général. Et moi en particulier et au premier chef" (Désir, p. 43). Le ton est plus neutre quand c'est le témoin qui assume le récit, comme dans "Ce n'était pas de nos affaires" où une voisine raconte l'histoire d'une femme nymphomane qui tue son propre enfant, sosie de son mari, parce que le mari ne lui faisait pas l'amour assez souvent. C'est un témoin anonyme qui se lamente du manque d'intervention des voisins ou des autorités:

On avait pourtant réussi à attirer l'attention des autorités concernées. Celles-ci avaient dépêché une jeune personne fort compréhensive et compétente, qui, après quelques visites, avait rapidement battu en retraite, impuissante. Elle ne pouvait guère conseiller à Carmen Trempe d'évacuer ses pulsions dans la prostitution ou la constitution d'un harem masculin, car ce n'est pas ce que l'on apprend traditionnellement aux Sciences sociales (Chiens, p. 30-31).

Malgré le ton humoristique et la critique implicite de la rectitude politique, le motif de la colère contre l'amant infidèle ou inattentif transmuée en haine de soi ou d'autrui se révèle plus troublant que la torture érotique décrite dans "Histoire de Q" de Dandurand, récit marqué, comme le dit bien von Flotow, par un moralisme prononcé ${ }^{16}$. Les nouvelles de Dé respirent souvent une forte ambiguité morale, comme le suggère la réaction de la voisine à l'égard de la nymphomanie de la femme meurtrière: "Parfois on la comprenait, parfois on l'excusait, mais le plus souvent on la condamnait. Comme s'il était si facile d'échapper à soimême" (Chiens, p. 28). Lire la violence passionnelle peut apporter la catharsis, mais Dé nous rappelle que la moralité de tout désir de vengeance est loin d'être nette.

Si les deux auteures partagent cet intérêt pour le crime passionnel, c'est Dandurand qui adapte le thème au récit policier.

16 Luise von Flotow, dans "Tenter l'érotique" (p. 69), parle de "fables moralisantes". 
Comme Dé, elle dépeint la femme justicière qui se venge ou venge les autres. Dans l'anthologie de nouvelles policières Saignant ou beurre noir?, sa nouvelle "Maîtresse des hautes ouvres" présente une femme qui trouve sa vocation en protégeant les faibles dans le métro. Elle tue un homme qui était en train de violer une femme, crève les yeux de celui qui allait pousser une femme obèse sur les voies du train et livre à la mort un homme qui voulait échapper à son sort de séropositif. Il s'agit pour elle d'un "lourd sacerdoce ${ }^{17}$ ". Cependant, c'est avec la création du détective Marc Mongeau que Dandurand entre dans le récit policier proprement dit. Mongeau figure à la fois dans les nouvelles policières de L'assassin de l'intérieur et comme personnage principal dans le roman La marquise ensanglantée ${ }^{18}$. Dans "L'Ex au max", il écoute d'une oreille compréhensive l'histoire du suspect Christophe qui décrit la façon dont son ex-amante Nina s'est arrangée pour le faire condamner pour sa mort à elle. Ici Dandurand marie le fantastique au policier: Mongeau et les lecteurs ne sauront jamais si Christophe a tué Nina ou si celle-ci s'est servi de pouvoirs surnaturels pour monter le coup contre lui. Peu importe, puisque Christophe, pénitent, s'avoue plus amoureux de Nina que jamais: "De son cachot Christophe contemple les étoiles. Il n'a pas assez aimé Nina. On l'a condamné au maximum, ce n'est pas payé trop cher. À présent, elle est toujours à ses côtés, ils bavardent à mivoix toute la journée, ils s'étreignent la nuit" (p. 33). La justice est rétablie; Mongeau ne peut plus rien pour lui. Dans "Le Salon des Coeurs perdus", Mongeau devient complice d'une femme justicière, une coiffeuse qui fait tuer par électrocution les amants qui maltraitaient ses clientes. Elle agit par pur esprit de justice sans avertir ses clientes de ses intentions. À la fin de son enquête, Mongeau se rend compte du rôle meurtrier de la coiffeuse mais il la comprend aussi et finit par s'éprendre d'elle au point de quitter son emploi pour devenir garçon-coiffeur dans son salon. Drôle de policier, Mongeau est surtout celui qui écoute, qui reconstitue les détails du crime et laisse aux femmes le rôle de justicier.

Du point de vue formel, il est à noter que le discours policier chez Dandurand se découpe parfois en passages datés. Dans "L'assassin de l'intérieur", une femme se tourne vers le journal

17 Collectif, Saignant ou beurre noir? 13 nouvelles policières, Québec, L'instant même, 1992, p. 23.

18 La marquise ensanglantée, Montréal, XYZ éditeur, 1996. 
82

intime pour essayer de déceler le mystère d'un intrus dans son appartement et d'une série de meurtres commis dans son entourage. C'est le journal Montréal-Matin, s'appuyant sur la reconstitution des événements par Mongeau, qui révèle que la femme a tendu un piège pour tuer l'intrus, alors que ce dernier n'est autre en fait qu'elle-même. Dans "L'ex au max", comme le remarque Gaétan Brulotte, Dandurand se sert de "l'ordre du calendrier et le temps de l'horloge pour rythmer le récit ${ }^{19}$ ". Surtout, la datation des événements marque la progression de l'enquête. L'effet de cette forme de journal de bord est tout autre que celui du journal imaginaire érotique de Dandurand. Si les tendances divergentes chez Dandurand et Dé se manifestent au niveau formel dans leur emploi distinctif du journal et du monologue intérieur, la thématique globale des deux nouvellières tend plutôt à converger.

\section{Des recueils de nouvelles au roman}

Artisanes renommées de la nouvelle, Dandurand et Dé ont publié plus récemment des romans qui révèlent une étroite parenté avec les recueils de nouvelles. Ce développement n'est guère étonnant puisque les critiques de la nouvelle ont souvent constaté un fort rapport entre le roman et le recueil homogène de nouvelles, reliés par des éléménts thématiques ou par la reprise des personnages ${ }^{20}$. Sourdes amours ${ }^{21}$, roman écrit par Dé, incorpore des éléments de sa nouvelle "Monomanie" (Chiens). On retrouve, par exemple, la même scène où la femme, cherchant une calculatrice dans la mallette de son mari, trouve un paquet de lettres d'une autre femme. De même, Dé emploie pour le roman cette même technique de monologue intérieur fortement saccadé que l'on a déjà remarquée dans certaines de ses nouvelles. Son

19 Gaétan Brulotte, "Formes de la nouvelle québécoise contemporaine", dans Lise Gauvin et Franca Marca-Falzoni (dir.), L'âge de la prose; romans et récits québécois des années 80, Rome, Bulzoni éditeur/Montréal, VLB éditeur, 1992, p. 81-82.

20 Voir à ce propos la distinction entre recueil homogène et recueil hétérogène chez André Carpentier et Denis Sauvé, "Le recueil de nouvelles", La nouvelle au Québec, Archives des Lettres canadiennes, tome IX, Montréal, Fides, 1996, p. 11-36. Carpentier rappelle l'idée de recueil homogène comme "quasiroman" chez François Ricard, "Le recueil", Études françaises, Montréal, vol. 12 , n ${ }^{\text {os }} 1-2$, avril 1976, p. 113-133.

21 Sourdes amours, Montréal, XYZ éditeur, 1993. 
roman le plus récent, Bonbeur, oiseau rare, porte la mention "roman pointilliste sous forme de haïku ${ }^{22}$ ". Très différent de la nouvelle par la forme, ce roman en haikku suggère néanmoins la même préférence pour les genres brefs. Dandurand semble aussi favoriser la brièveté avec son roman Un cour qui craque, le découpage en passages d'un journal imaginaire brisant la continuité $\mathrm{du}$ roman ${ }^{23}$. Les repères bibliographiques de son deuxième roman, La salle d'attente ${ }^{24}$, étiqueté "roman miniature", révèlent que certaines parties du roman avaient déjà été publiées ailleurs comme récits indépendants. Son roman le plus récent, La marquise ensanglantée, s'éloigne de la nouvelle par sa forme plus développée mais met en vedette deux personnages de nouvelles antérieures: le détective Marc Mongeau, complice des femmes vengeresses, et Jeanne Couteau, l'ex-sorcière, recyclée en actrice et en peintre.

Comme toute analyse implique un degré de simplification, cet aperçu des nouvelles de ces écrivaines jumelles laisse de côté nécessairement bien des aspects importants de leur ouvre dont, par exemple, le thème du nationalisme québécois. Ludiques, sensuelles, intenses, les nouvelles d'Anne Dandurand et de Claire Dé livrent une vision intime de la femme, une célébration des pulsions érotiques au féminin, relevant d'une solidarité féministe qui dépasse celle de la gémellité.

22 Bonheur, oiseau rare, Montréal, XYZ éditeur, 1996.

23 Un cour qui craque (journal imaginaire), Montréal, VLB éditeur/Messidor, 1990.

24 La salle d'attente, Montréal, XYZ éditeur, 1994. 